
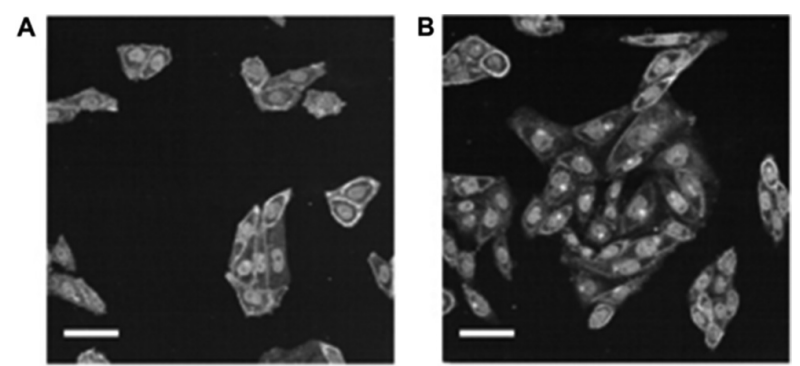

Abstract BS16 Figure 1 Triple fluorescence confocal images of $\mathrm{CHO}$ K1 cells stably expressing the apelin receptor. Apelin-488 shown in green, nuclear staining in blue by Hoechst 33342 and membrane staining in orange by a wheat germ agglutinin-AlexaFluor-594 conjugate. when treated with the fluorescent ligand for 30secs (Fig 1A) an overlap is seen between the membrane and apelin-488, shown in yellow. After 60mins incubation with ligand (Fig 1B), less receptor is seen at the membrane and is intead localised intracellularly. Scale bar represents $50 \mu \mathrm{m}$

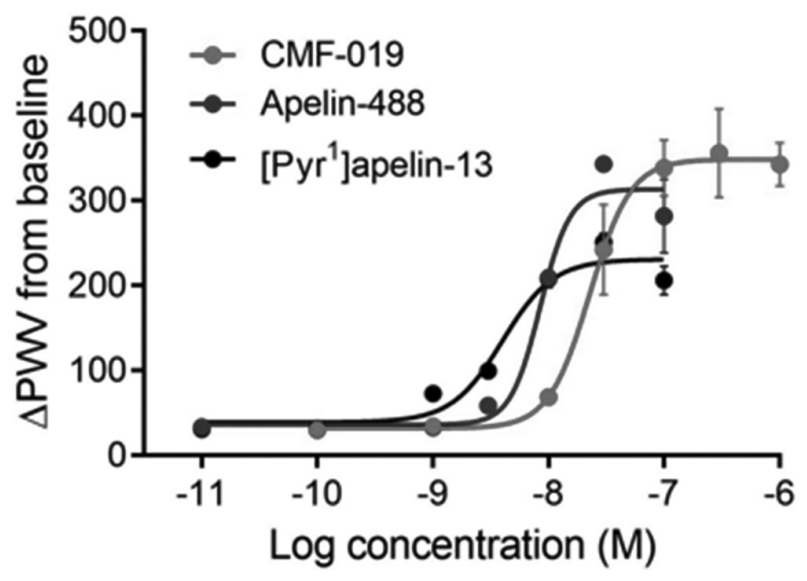

Abstract BS16 Figure 2 Dynamic mass redistribution assay showing activity of apelin receptor ligands, CMF-019, Apelin-488 and [Pyr ${ }^{1}$ ] apelin-13 in CHO-K1 cells stably expressing the apelin receptor

Conclusion Apelin-488 shows specific binding to the apelin receptor while retaining activity in a dynamic mass redistribution and binding assays. This work provides evidence for apelin-488 as a novel fluorescent tool for assessing internalisation of the apelin receptor following receptor binding. A new method for studying apelin receptor internalisation is particularly interesting due to the implication of apelin receptor desensitisation in pulmonary arterial hypertension, mediated by arrestin and internalisation pathways. Additionally, apelin-488 may provide insight into binding and receptor trafficking in mutated apelin receptors previously identified in patients with rare cardiovascular diseases. As such, apelin-488 and other fluorescent ligands may help streamline development of drugs that could be beneficial in clinical settings.

Conflict of interest none

\section{BS17 CITED2 REGULATES TROPHOBLAST GLYCOPROTEIN EXPRESSION IN THE ADVENTITIAL PERICYTE AND MIGRATION}

William Cathery ${ }^{*}$ Eva Jover, Paolo Madeddu. University of Bristol

\subsection{6/heartjnl-2019-BCS.180}

Introduction Hypoxia induces migration in adventitial pericytes (APCs), conferring them with therapeutic potential towards the acquisition of a proangiogenic pericyte-like phenotype. Hypoxic activation of HIF1alpha can regulate epithelial-tomesenchymal transitions (EMT), including the mesenchymal-topericyte transition. TPBG is an EMT marker which is associated with migration. CITED2 regulates the HIF1alpha hypoxia-induced genes, mainly under hypoxic conditions. We aim to demonstrate the role of TPBG on hypoxic-dependent migration of APCs and to identify the mechanism underpinning TPBG transcription.

Methods \& Results: CD34/NG2 positive APCs were isolated from saphenous vein leftovers (from CABG). APCs were conditioned for 24,48 and $72 \mathrm{~h}$ under normoxia or hypoxia $(2 \%$ O2). Hypoxia induces the mRNA expression of classic EMT markers (SNAIL1/2, ZEB2, TIMP1/2) and TPBG $(1.97 \pm 0.27$ mRNA fold change after vs. normoxia; $2.63 \pm 0.62$ protein fold change vs. normoxia, both $\mathrm{p}<0.05)$. TPBG loss-of-function (LOF) by siRNA or gain-of-function (GOF) by TPBG overexpression were used to validate the role of TPBG in migration. LOF resulted in reduced scratch assay migration $(0.67 \pm 0.03, \mathrm{p}<0.01)$, whilst GOF resulted in increased migration $(5.40 \pm 0.60, \mathrm{p}<0.01)$. CITED2 was found progressively down-regulated after $48 \mathrm{~h}$ of hypoxic conditioning at the transcript level but not at the protein level $(1.41 \pm 0.07$-fold change after $48 \mathrm{~h}$ of hypoxia vs. normoxia, $\mathrm{p}<0.05$ ). CITED2/TPBG interaction was further studied by over-expressing CITED2. ChiP analysis evidenced the binding of CITED2 to TPBG at the TSS $-1985 /-1836$ and $+482 /+555$ under normoxia, and that was considerably enhanced by hypoxia (both $\mathrm{p}<0.05)$. LOF experiments were performed in hypoxic APCs by silencing CITED2 (70nM). A pool of 4 scrambled siRNAs was used as control in parallel. Knocking-down effect was validated by $\mathrm{qPCR}$ in 4 APC lines showing $0.30 \pm 0.13$-fold change expression of the CITED2 transcript, $\mathrm{p}<0.001$ (qPCR, 2-DDCT method). That was confirmed by ICC showing a nuclear location with a modest but significant reduction of immunoreactive CITED2. CITED2 LOF was associated with a significant down-regulation of TPBG at protein levels after 48 or $72 \mathrm{~h}$ depending on the cell line and by an overall $0.78 \pm$ $0.10, p=0.02$ compared to siRNA control. Wound healing experiments additionally showed that silencing of CITED2 was associated with a lower migration profile in the APC $(0.70 \pm$ $0.05, \mathrm{p}<0.01)$.

Conclusion TPBG plays a crucial role in APC migration. CITED2 significantly affects TPBG expression in hypoxic APCs and thus hypoxia-induced migration in APCs. Discordant protein and transcript levels suggest a tight regulation of the CITED2 stability, pinpointing an important role of CITED2 in the APC. These findings might be relevant for regenerative medicine applications due to the association among APC migration and TPBG expression.

Conflict of interest $\mathrm{N} / \mathrm{a}$ 